\title{
DIFFEOMORPHISMS OBTAINED FROM ENDOMORPHISMS
}

\author{
BY
}

\section{LOUIS BLOCK $\left({ }^{1}\right)$}

\begin{abstract}
It is shown that if $f$ is a differentiable map of a compact manifold, and the singularities of $f$ satisfy a certain condition, then there is a diffeomorphism (of a different manifold) whose orbit structure is closely related to that of $f$. This theorem is then used to extend several results on the orbit structure of diffeomorphisms to the noninvertible case.
\end{abstract}

1. Statement of results. Let $M$ denote a compact manifold without boundary, and $\operatorname{End}(M)$ denote the space of $C^{1}$ maps of $M$ into itself, with the $C^{1}$ topology. For $f \in \operatorname{End}(M)$ let $\Omega(f)$ denote the nonwandering set of $f$, which we define in $\S 2 . \Omega(f)$ is a closed set and $f(\Omega(f)) \subset \Omega(f)$. We use the notation $\stackrel{\lim }{\leftarrow}(f, \Omega(f))$ to denote the inverse limit of the map $f: \Omega(f) \rightarrow \Omega(f)$, as defined in $\S 2$.

Our main results are the following (see $\S 2$ for definitions):

Let $f \in \operatorname{End}(M)$ with all singularities of $f$ in the stable manifolds of orbits of sinks.

THEOREM A. There is a diffeomorphism $\hat{f}$ of a tubular neighborhood of $M$ into itself such that $\hat{f} \mid \Omega(\hat{f})$ is topologically conjugate to $\lim (f, \Omega(f))$. Furthermore $f$ satisfies Axiom $\mathrm{A}$ if and only if $\hat{f}$ does.

THEOREM B. $f(\Omega(f))=\Omega(f)$.

THEOREM C. If $f$ satisfies Axiom $\mathrm{A}$, then $f$ has a spectral decomposition.

THEOREM D. Suppose $f$ satisfies Axiom A, and $\Omega(f)$ consists of expanding basic sets together with finitely many sinks. Then $f$ is $\Omega$-stable.

Theorem A generalizes a theorem and construction of Williams [11]. Also if $f$ is an expanding map, the diffeomorphism $\hat{f}$ is just the $D E$ map of Smale [9]. Using Theorem A and its proof, we can prove certain properties of endomorphisms (satisfying the hypothesis of Theorem A) from the corresponding properties of

Received by the editors January $22,1975$.

AMS (MOS) subject classifications (1970). Primary 58F15; Secondary 58C25.

Key words and phrases. Endomorphism, diffeomorphism, nonwandering set, hyperbolic structure, singularity, inverse limit.

(1) Some of the results of this paper appeared in the author's thesis, which was completed at Northwestern University and the Institut des Hautes Études Scientifiques under the supervision of $\mathbf{R}$. F. Williams. 
diffeomorphisms. Theorems $\mathrm{B}, \mathrm{C}$ and $\mathrm{D}$ are examples of this. These give partial answers to questions raised by Shub in [8], concerning generalizations of theorems on the orbit structure of diffeomorphisms to the endomorphism case (i.e. the noninvertible case). See [9] for an introduction to the theory for diffeomorphsims, and [1], [2], [3], [4] , [6] and [8] for some examples of results in the theory for endomorphisms.

In connection with Theorem $B$, we give an example in $\$ 4$ of an endomorphism of the circle such that $f(\Omega(f)) \neq \Omega(f)$. Of course, $f(\Omega(f))=\Omega(f)$ for any diffeomorphism.

The proof of Theorem $C$ uses Smale's spectral decomposition theorem for Axiom A diffeomorphisms [9], and the proof of Theorem D uses Smale's $\Omega$-stability theorem for diffeomorphisms [10].

Theorem D contains the following known results:

THEOREM (JACOBSON [6]). Let $f \in \operatorname{End}\left(S^{1}\right)$ with all singularities of $f$ in the stable manifolds of sinks, and suppose $f$ satisfies Axiom A. Then $f$ is $\Omega$-stable.

TheOREM (SHUB [8]). An expanding endomorphism of a compact manifold is structurally stable.

To obtain the latter theorem from Theorem D, we note that for an expanding endomorphism every point is nonwandering.

Theorem A may also be used to give examples of diffeomorphisms. Guckenheimer in [4] uses this idea to give an example of a basic set which is not locally the product of a Cantor set and a manifold. In fact Guckenheimer states the following proposition (Propositon 6.1 of [4]) which is similar to Theorem A.

Proposition. Suppose $f: M^{n} \rightarrow M^{n}$ is a map such that $f \mid \Omega(f)$ is a local homeomorphism. Then $\exists g \in \operatorname{Diff}\left(S^{2 n+2}\right)$ such that $\Omega(g)$ contains components homeomorphic to the components of $\lim (f, \Omega(f))$.

However, Guckenheimer's proof of this proposition is incorrect. See $\S 6$ for an example and further discussion.

The example in $\S 6$ shows that Guckenheimer's proof does not work when singularities such as folds are present. However, his proof is valid when $f$ is an open mapping, and in his example $f$ is an open mapping, so the example is certainly correct.

Clearly the hypothesis of Proposition 6.1 is more general than the hypothesis of Theorem A, so the validity of this proposition remains an open question.

I would like to thank J. Franks, R. Clark Robinson and R. F. Williams for helpful conversations about this work.

2. Preliminary definitions. Fix $f \in \operatorname{End}(M)$. A point $x \in M$ is said to be wandering if there is a neighborhood 0 of $x$ such that $f^{n}(0) \cap 0=\varnothing$ for all $n>0$ 
(here $f^{n}$ is defined inductively by $f^{1}=f$ and $f^{n}=f \circ f^{n-1}$ ). The set of points which are not wandering is called the nonwandering set and denoted by $\Omega(f)$.

A point $x \in M$ is called a singularity of $f$ if $D f(x)$ (the derivative of $f$ at $x$ ) is not an isomorphism, or equivalently if $f$ is not a local diffeomorphism at $x$.

A point $x \in M$ is called a periodic point of $f$ if $f^{n}(x)=x$ for some $n>0$. The minimum of $\left\{n: f^{n}(x)=x\right\}$ is called the period of $x$. We let $\operatorname{per}(f)$ denote the set of periodic points of $f$. A periodic point $x$ of period $n$ is called a sink (or contracting periodic point) if all eigenvalues of $D f^{n}(x)$ have absolute value less than one.

We say $f$ satisfies Axiom A if $\overline{\operatorname{per}(f)}=\Omega(f)$ and $\Omega(f)$ has a hyperbolic structure. The second condition means that there are constants $0<\lambda<1<\gamma$, and $c>0$, and $k>0$, and a continuous splitting of the tangent bundle of $M$ restricted to $\Omega(f), T_{\Omega(f)}(M)=E^{s} \oplus E^{u}$ such that $D f$ preserves the splitting and $\left\|D f^{n}(x)\right\|<c \lambda^{n}\|x\|$ for $x \in E^{s}$, while $\left\|D f^{n}(x)\right\|>k \gamma^{n}\|x\|$ for $x \in E^{u}$. Here \|I $\|$ denotes any Riemannian metric on $M$, and the definition is independent of the choice of Riemannian metric (see [9]).

If $x$ is a sink of $f$, the stable manifold of $x$ is denoted $W^{s}(x)$, and consists of those points $y \in M$ such that $x$ is a limit point of $\operatorname{orb}(y)$. Here

$$
\operatorname{orb}(y)=\left\{f^{n}(y): n \geqslant 0\right\} .
$$

Note that $W^{s}(x)$ is an open subset of $M$.

We say $f$ has a spectral decomposition if we can write $\Omega(f)$ as a finite union of pairwise disjoint closed sets, $\Omega(f)=\Omega_{1} \cup \cdots \cup \Omega_{n}$ with $f\left(\Omega_{i}\right)=\Omega_{i}$ for $i=1, \ldots, n$, such that each $\Omega_{i}$ contains a point whose orbit is dense in $\Omega_{i}$. The $\Omega_{i}$ are called basic sets.

Any two maps $f: K \rightarrow K$ and $g: L \rightarrow L$ of topological spaces are said to be topologically conjugate if there is a homeomorphism $h: K \rightarrow L$ such that $h(f(x))=g(h(x))$ for all $x \in K$. We say $f \in \operatorname{End}(M)$ is $\Omega$-stable if there is a neighborhood $N(f)$ in $\operatorname{End}(M)$ such that any $g \in N(f)$ is $\Omega$-conjugate to $f$, i.e. $f \mid \Omega(f)$ and $g \mid \Omega(g)$ are topologically conjugate.

Finally let $(K, d)$ be a compact metric space and $f: K \rightarrow K$ a continuous map. We define $\lim _{\longleftarrow}(f, K)$ to be the set of sequences $\left(a_{0}, a_{1}, a_{2}, \ldots\right)$ such that for each $i>0$, we have $a_{i} \in K$ and $f\left(a_{i}\right)=a_{i-1}$. We put a metric $\hat{d}$ on $\lim _{\leftarrow}(f, K)$ defined by:

$$
\hat{d}\left(\left(a_{0}, a_{1}, a_{2}, \ldots\right),\left(b_{0}, b_{1}, b_{2}, \ldots\right)\right)=\sum_{i=0}^{\infty} d\left(a_{i}, b_{i}\right) 2^{-i} .
$$

$f$ induces a homeomorphism $F$ on $\lim _{\longleftarrow}(f, K)$ defined by

$$
F\left(a_{0}, a_{1}, a_{2}, \ldots\right)=\left(f\left(a_{0}\right), f\left(a_{1}\right), f\left(a_{2}\right), \ldots\right)=\left(f\left(a_{0}\right), a_{0}, a_{1}, \ldots\right) .
$$

We will (as in the statement of Theorem $\mathrm{A}$ ) think of $\lim _{(}(f, K)$ as the space, together with the homeomorphism induced by $f$. 


\section{Proof of Theorem A.}

LEMMA 1. Let $(K, d)$ be a compact metric space and $f: K \rightarrow K$ a continuous map. Suppose $\overline{\operatorname{per}(f)}=K$. Then $\overline{\operatorname{per}(F)}=\lim (f, K)$, where $F$ is the homeomorphism induced by $f$ on $\lim (f, K)$.

Proof. Let $\left(a_{0}, a_{1}, a_{2}, \ldots\right) \in \lim (f, K)$. Let $\epsilon>0$. Pick $n$ so large that $\sum_{i=n}^{\infty} D 2^{-i}<1 / 2 \epsilon$, where $D$ is the diameter of $K$. Pick $b \in \operatorname{per}(f)$ close enough to $a_{n}$ to insure that

$$
\sum_{i=0}^{n} d\left(f^{i}(b), f^{i}\left(a_{n}\right)\right) 2^{(i-n)}<\frac{1}{2} \epsilon
$$

Then $\left(f^{n}(b), f^{n-1}(b), \ldots, f(b), b, f^{-1}(b), f^{-2}(b), \ldots\right)$, where $f^{-1}$ is taken in $\operatorname{orb}(b)$, is a periodic point of $F$ within $\epsilon$ of $\left(a_{0}, a_{1}, a_{2}, \ldots\right)$ Q.E.D.

We now prove Theorem A. It may be helpful for the reader to see the example in $\S 6$, to help visualize the construction.

TheOREM A. Let $f \in \operatorname{End}(M)$ with all singularities of $f$ in the stable manifolds of sinks. There is a diffeomorphism $\hat{f}$ of a tubular neighborhood of $M$, into itself such that $\hat{f} \mid \Omega(\hat{f})$ is topologically conjugate to $\lim (f, \Omega(f))$. Furthermore, $f$ satisfies Axiom A if and only if $\hat{f}$ does.

Proof. Since the singularity set is closed, it must be contained in the stable manifolds of finitely many sinks $s_{1}, s_{2}, \ldots, s_{p}$. Let $n$ be the dimension of $M$ and $i: M \rightarrow S^{2 n+2}$ be an embedding. For simplicity we may assume $i$ is the inclusion, i.e., $M \subset S^{2 n+2}$. Let $N$ be a tubular neighborhood of $M$ in $S^{2 n+2}$. Perturb $f$ to an embedding $f_{1}: M \rightarrow N$ such that $\pi\left(f_{1}(x)\right)=f(x)$, except possibly on a closed subset of $\bigcup_{i=1}^{p} W^{s}\left(s_{i}\right)$, where $\pi: N \rightarrow M$ is the projection. It follows from the dimensions of $M$ and $S^{2 n+2}$ that $f_{1}$ is isotopic to the inclusion $i$. Hence by the isotopy extension theorem (Theorem 5.8 of [7]) $f_{1}$ may be extended to a diffeomorphism $f_{2}$ of $S^{2 n+2}$. Let $\hat{f}=f_{2} \mid N$. We can modify $f_{2}$ so that $\hat{f}$ maps $N$ into itself and:

1. $\hat{f}$ is a contraction on each fiber of $N$ over $M$, and $\hat{f}$ has sinks $\hat{s}_{1}, \hat{s}_{2}, \ldots$, $\hat{s}_{p}$ corresponding to $s_{1}, s_{2}, \ldots, s_{p}$.

2. $\hat{f}$ is fiber preserving except possibly on a closed set $B$, such that $B$ consists of a closed subset $B_{0}$ of $M$ and its fibers,

$$
\bigcup_{n \geqslant 0}(\hat{f})^{n}(B) \subset \bigcup_{i=1}^{p} W^{s}\left(\hat{s_{i}}\right) \text { and } \bigcup_{n \geq 0} f^{n}\left(B_{0}\right) \subset \bigcup_{i=1}^{p} W^{s}\left(s_{i}\right) \text {. }
$$

Let

$$
\lambda_{1}=d\left(\bigcup_{n \geq 0}(\hat{f})^{n}(B), N-\bigcup_{i=1}^{p} W^{s}\left(\hat{s}_{i}\right)\right)
$$


and

$$
\lambda_{2}=d\left(\bigcup_{n \geq 0} f^{n}\left(B_{0}\right), M-\bigcup_{i=1}^{p} W^{s}\left(s_{i}\right)\right) .
$$

Let $\lambda=\min \left\{\lambda_{1}, \lambda_{2}\right\}$. Then $\lambda>0$. Since by construction for $i=1, \ldots, p$ we have a correspondence between $s_{i}$ and $\hat{s}_{i}$, it suffices to consider the parts of $\Omega(f)$ and $\Omega(\hat{f})$ not in $W^{s}\left(s_{i}\right)$ or $W^{s}\left(\hat{s}_{i}\right)$ respectively, and we adopt this convention for the remainder of this proof.

Step 1. $\pi(\Omega(\hat{f}))=\Omega(f)$.

First we show $\pi(\Omega(\hat{f})) \supset \Omega(f)$. Let $x \in \Omega(f)$. We must show there is a point in $\Omega(\hat{f})$ in fib $(x)$, the fiber over $x$. Suppose each point in fib $(x)$ is not in $\Omega(\hat{f})$. Then each point in fib $(x)$ has a neighborhood such that no iterate (with respect to $\hat{f})$ of the neighborhood intersects itself. Since fib $(x)$ is compact we can take a finite subcover, $\left\{U_{1}, \ldots, U_{m}\right\}$ of these neighborhoods. $\exists \epsilon<\lambda$ such that if $d(x, y) \leqslant \epsilon$ then fib $(y) \subset U_{1} \cup U_{2} \cup \cdots \cup U_{m}$. By choosing $\epsilon$ smaller if necessary, we may assume for $i=1, \ldots, m$ that $\operatorname{fib}(y) \cap U_{i} \neq \varnothing$ if $d(x, y) \leqslant \epsilon$. Let $K=\bigcup$ fib $(y)$ where the union is taken over all $y$ with $d(x, y) \leqslant \epsilon$. Then $K$ is compact and $\left\{U_{1}, \ldots, U_{m}\right\}$ is a cover of $K$. Hence $\exists \delta>0$ such that any subset of $K$, of diameter less than $\delta$ is contained in some $U_{j}$.

Pick $N_{2}$ so large that if $n>N_{2}$, and the first $n$ iterates of $z$ are not in $B$, then $(\hat{f})^{n}$ (fib $\left.(z)\right)$ has diameter less than $\delta$. Since $x \in \Omega(f), \exists y \in M$, with $d(x, y) \leqslant \epsilon$, and an $n>N_{2}$ such that $d\left(x, f^{n}(y)\right) \leqslant \epsilon$. Since $\epsilon<\lambda$, none of the first $n$ iterates of $y$ are in $B$, so $(\hat{f})^{n}$ is fiber preserving on fib $(y)$. By choice of $N_{2}$ and $\delta,(\hat{f})^{n}$ maps fib $(y)$ into one of $\left\{U_{1}, \ldots, U_{m}\right\}$ say $U_{j}$. Then $(\hat{f})^{n}\left(U_{j}\right)$ $\cap\left(U_{j}\right) \neq \varnothing$. This contradicts the choice of the $U_{i}$ 's. Hence $\pi(\Omega(\hat{f})) \supset \Omega(f)$.

We now show $\Omega(f) \supset \pi(\Omega(\hat{f}))$. We must show that if a point in fib $(y)$ is in $\Omega(\hat{f})$ then $y \in \Omega(f)$. Let $z \in$ fib $(y)$ be in $\Omega(\hat{f})$. Let $\epsilon>0$. Pick $\delta>0$ such that if $d(q, z)<\delta$ then $d(\pi(q), y)<\epsilon$. Without loss of generality we may assume that $\lambda>\max \{\epsilon, \delta\} . \exists q \in N$ such that $d(q, z)<\delta$ with $d\left((\hat{f})^{n}(q), z\right)<\delta$ for some $n>0$. Hence fib $(\pi(q))$ is mapped by $(\hat{f})^{n}$ in a fiber preserving way. Hence $d(\pi(q), y)<\epsilon$ and $d\left(f^{n}(\pi(q)), y\right)<\epsilon$. Since $\epsilon$ is arbitrary, $y \in \Omega(f)$. Hence $\Omega(f) \supset \pi(\Omega(\hat{f}))$.

Step 2. $\hat{f} \mid \Omega(\hat{f})$ is topologically conjugate to $\lim (f, \Omega(f))$.

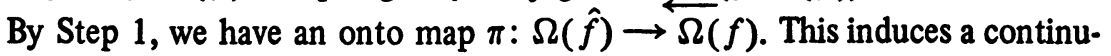
ous map $p: \lim (\hat{f}, \Omega(\hat{f})) \rightarrow \lim (f, \Omega(f))$ defined by $p\left(a_{1}, a_{2}, \ldots\right)=$ $\left(\pi\left(a_{1}\right), \pi\left(a_{2}\right), \ldots\right)$. First we show $p$ is $1-1$. Suppose $p\left(a_{1}, a_{2}, \ldots\right)=$ $p\left(b_{1}, b_{2}, \ldots\right)$ with $a_{j} \neq b_{j}$ for some $j$. Then $a_{i}$ and $b_{i}$ are on the same fibers for each $i$. Pick $n$ so large that diameter $\left((\hat{f})^{n}(f i b(x))\right)<d\left(a_{j}, b_{j}\right)$, if $x$ and its iterates are in the part of $M$, where $\hat{f}$ is fiber preserving. Then $a_{j+n}$ and $b_{j+n}$ are on the same fiber of a point in $\Omega(f)$, so $d\left((\hat{f})^{n}\left(a_{j+n}\right),(\hat{f})^{n}\left(b_{j+n}\right)\right)<d\left(a_{j}, b_{j}\right)$. This is a contradiction since $(\hat{f})^{n}\left(a_{j+n}\right)=a_{j}$ and $(\hat{f})^{n}\left(b_{j+n}\right)=b_{j}$. Hence $p$ is 1-1. 
Next we show $p$ is onto. Let $\left(a_{0}, a_{1}, \ldots\right) \in \lim (f, \Omega(f))$. Notice $(\hat{f})^{i}\left(\pi^{-1}\left(a_{i}\right)\right)$ is a decreasing sequence of nonempty compact sets. Hence $\bigcap_{n>0}(\hat{f})^{n}\left(\pi^{-1}\left(a_{n}\right)\right)$ is nonempty, and if $b_{0}$ is in this intersection then $p\left(b_{0},(\hat{f})^{-1}\left(b_{0}\right),(\hat{f})^{-2}\left(b_{0}\right), \ldots\right)=\left(a_{0}, a_{1}, a_{2}, \ldots\right)$. Hence $p$ is onto. It follows that $p$ is a homeomorphism. Since $\pi$ is fiber preserving, $p$ is a conjugacy between $\lim (\hat{f}, \Omega(\hat{f}))$ and $\lim (f, \Omega(f))$. But $\hat{f}$ is a homeomorphism on $\Omega(\hat{f})$ so $\underset{\lim }{\longleftarrow}(\hat{f}, \bar{\Omega}(\hat{f}))$ is topologically conjugate to $\hat{f} \mid \Omega(\hat{f})$.

Step 3. $f$ satisfies Axiom $\mathrm{A}$ if and only if $\hat{f}$ does.

It is clear by the construction of $\hat{f}$ that $f$ has a hyperbolic structure if and only if $\hat{f}$ does.

If $\overline{\operatorname{per}(f)}=\Omega(f)$, then $\overline{\operatorname{per}(\hat{f})}=\Omega(\hat{f})$ by Lemma 1 and Step 2 .

Suppose $\overline{\operatorname{per}(\hat{f})}=\Omega(\hat{f})$. Let $y \in(\Omega(f)-\operatorname{per}(f))$. By Step $1, \exists Z \in$ $\Omega(\hat{f})$ in fib $(y)$. Let $\left(e_{i}\right)$ be a sequence of periodic points of $\hat{f}$ approaching $Z$. Since $\hat{f}$ is fiber preserving on fib $\left(\pi\left(e_{i}\right)\right)$ for each $i, \pi\left(e_{i}\right)$ is a sequence of periodic points of $f$ approaching $y$. Hence $\overline{\operatorname{per}(f)}=\Omega(f)$. Q.E.D.

\section{Proof of Theorem B.}

THEOREM B. Let $f \in \operatorname{End}(M)$ with all singularities of $f$ in the stable manifolds of sinks. Then $f(\Omega(f))=\Omega(f)$.

Proof. Let $\hat{f}$ be the diffeomorphism of a tubular neighborhood $N$ of $M$, constructed in Theorem A, and let $\pi$ be the projection $\pi: N \rightarrow M$. By the proof of Theorem A, we have the following commutative diagram

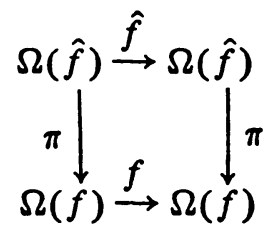

where $\pi$ maps $\Omega(\hat{f})$ onto $\Omega(f)$. Since $\hat{f}$ is one-to-one, it follows that $\hat{f}$ maps $\Omega(\hat{f})$ onto $\Omega(\hat{f})$. Hence by the diagram $f$ maps $\Omega(f)$ onto $\Omega(f)$. Q.E.D.

We conclude this section by giving an example of a $\operatorname{map} f \in \operatorname{End}\left(S^{1}\right)$ such that $f(\Omega(f)) \neq \Omega(f)$. The example is shown in Figure 1. Here $e$ is an expanding fixed point of $f, c$ is a contracting fixed point of $f, s_{1}, s_{2}, s_{3}$ and $s_{4}$ are singularities, and the crucial condition is that $f^{2}\left(s_{1}\right)=e$.

Note that $s_{1}$ is wandering. This is true because if 0 is a small open interval about $s_{1}, f(0)$ is a half open interval with right endpoint $f\left(s_{1}\right)$, and $f^{2}(0)$ is a half open interval with right endpoint $e$. Thus all points in 0 wander to $c$. However $f\left(s_{1}\right) \in \Omega(f)$ because if $U$ is an open interval about $f\left(s_{1}\right), f(U)$ will be an open interval containing $e$, so some iterate of $U$ will intersect $U$ (since $e$ is expanding), Also $f\left(s_{1}\right)$ is the image only of the point $s_{1}$. Hence $f\left(s_{1}\right) \in \Omega(f)$ but $f\left(s_{1}\right) \notin f(\Omega(f))$. 


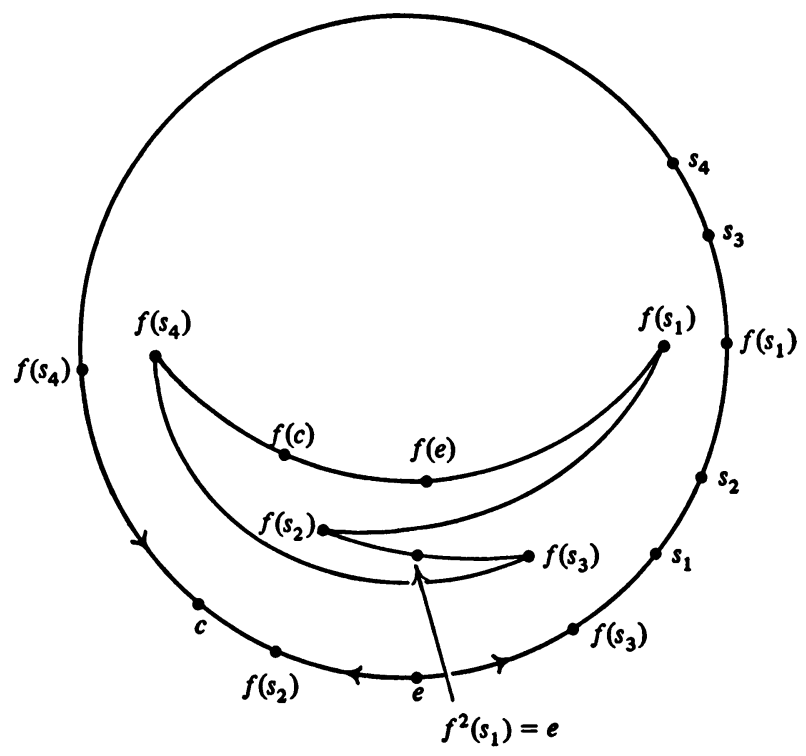

FIGURE 1

5. Proof of Theorems $\mathbf{C}$ and D. In the proof of Theorem $\mathbf{C}$ we use Smale's spectral decomposition theorem for diffeomorphisms, which states that if $f$ is a diffeomorphism of a compact manifold (without boundary) onto itself, and $f$ satisfies Axiom A, then $f$ has a spectral decomposition (see [9]). This theorem is valid for a diffeomorphism of a tubular neighborhood into itself (such as the one constructed in Theorem A), because such a diffeomorphism can be extended to a diffeomorphism of a sphere (of suitable dimension) onto itself.

Theorem C. Let $f \in \operatorname{End}(M)$ satisfy Axiom A, with all singularities of $f$ in the stable manifolds of sinks. Then $f$ has a spectral decomposition.

Proof. Let $\hat{f}$ be as in Theorem $A$ and let $\Omega(\hat{f})=\hat{\Omega}_{1} \cup \cdots \cup \hat{\Omega}_{n}$ be the spectral decomposition for $\hat{f}$. Let $\Omega_{i}=\pi\left(\hat{\Omega}_{i}\right)$ for $i=1, \ldots, n$. We will show the $\Omega_{i}$ 's give a spectral decomposition for $f$. Since $\pi$ maps $\Omega(\hat{f})$ onto $\Omega(f)$, we have $\Omega(f)=\Omega_{1} \cup \cdots \cup \Omega_{n}$. Also, if $a_{i}$ has a dense orbit in $\hat{\Omega}_{i}$, then $\pi\left(a_{i}\right)$ has a dense orbit in $\Omega_{i}$. Since $\hat{f}$ is fiber preserving on $\Omega(\hat{f}), f\left(\Omega_{i}\right) \subset \Omega_{i} \forall_{i}$. By Theorem B, $f\left(\Omega_{i}\right)=\Omega_{i} \forall_{i}$.

It remains to show that if $i \neq j$, then $\Omega_{i} \cap \Omega_{j}=\varnothing$. Let $x \in \Omega_{i} \cap \Omega_{j}$. Then $\exists y \in \hat{\Omega}_{i}, z \in \hat{\Omega}_{j}$ such that $\pi(y)=\pi(z)=x$. Since $\hat{f}$ is a contraction on fib $(x), d\left((\hat{f})^{n}(y),(\hat{f})^{n}(z)\right) \rightarrow 0$ as $n \rightarrow \infty$. This is a contradiction since $(\hat{f})^{n}(y) \in \hat{\Omega}_{i}, \forall n \geqslant 0$, and $(\hat{f})^{n}(z) \in \hat{\Omega}_{j}, \forall n \geqslant 0$, and $\hat{\Omega}_{i}$ and $\hat{\Omega}_{j}$ are disjoint compact sets. Q.E.D.

Let $f \in \operatorname{End}(M)$ satisfy Axiom A with all singularities of $f$ in the stable manifolds of sinks. Let $\Omega(f)=\Omega_{1} \cup \cdots \cup \Omega_{n}$ be the spectral decomposition 
of $\Omega(f)$. We define $W^{s}\left(\Omega_{i}\right)$ and $W^{u}\left(\Omega_{i}\right)$ by saying $x \in W^{s}\left(\Omega_{i}\right)$ if $f^{n}(x) \rightarrow$ $\Omega_{i}$ as $n \rightarrow \infty$, and $x \in W^{u}\left(\Omega_{i}\right)$ if there is a sequence $\left(x^{-n}\right)$, with $x^{0}=x$ and $f\left(x^{-i}\right)=x^{-i+1} \forall i \geqslant 1$, such that $x^{-n} \rightarrow \Omega_{i}$ as $n \rightarrow \infty$. We write $\Omega_{i}<\Omega_{j}$ if $W^{u}\left(\Omega_{j}\right) \cap W^{s}\left(\Omega_{i}\right) \neq \varnothing$. We say a set $\left\{\Omega_{i_{1}}, \ldots, \Omega_{i_{r}}\right\}$ of distinct $\Omega_{i}$, forms a cycle if $r>1$ and $\Omega_{i_{1}}<\Omega_{i_{2}}<\cdots<\Omega_{i_{r}}<\Omega_{i_{1}} \cdot f$ is said to satisfy the nocycle property if there are no cycles.

LEMMA 2. Let $f$ and $\hat{f}$ be as in Theorem A. $f$ satisfies the no-cycle property $\Longleftrightarrow \hat{f}$ does.

Proof. We first remark that if $\Omega_{k}$ consists of the orbit of a sink, then $\Omega_{k}$ cannot be a part of a cycle. Hence we may consider only those $\Omega_{k}$ which do not consist of the orbit of a sink.

$\Leftrightarrow$ It suffices to show that if $\hat{\Omega}_{i}<\hat{\Omega}_{j}$ then $\Omega_{i}<\Omega_{j}$. Let $x \in W^{u}\left(\hat{\Omega}_{j}\right) \cap$ $W^{s}\left(\hat{\Omega}_{i}\right)$. Since $(\hat{f})^{n}(x) \rightarrow \hat{\Omega}_{j}$, orb $(x) \cap B=\varnothing$, where $B$ is as in Theorem $\mathbf{A}$. Hence $\hat{f}$ is fiber preserving on orb $(x)$, including negative iterates of $x$. Thus $\pi(x) \in$ $W^{u}\left(\Omega_{j}\right) \cap W^{s}\left(\Omega_{i}\right)$.

$(\Leftrightarrow)$ It suffices to show that if $\Omega_{i}<\Omega_{j}$ then $\hat{\Omega}_{i}<\hat{\Omega}_{j}$. Let $x \in W^{u}\left(\Omega_{j}\right) \cap$ $W^{s}\left(\Omega_{i}\right)$. Then $\hat{f}$ is fiber preserving on orb $(x)$ including negative iterates of $x$. Let $\left(x^{-n}\right)$ be a sequence of points with $x^{0}=x$, and $f\left(x^{-n}\right)=x^{-n+1}$, such that $x^{-n} \rightarrow \Omega_{j}$ as $n \rightarrow \infty$. From the proof of Theorem $A, \bigcap_{n \geq 0}(\hat{f})^{n}\left(f i b\left(x^{-n}\right)\right)$ consists of a single point $y$ in fib $(x)$. Then the sequence $\left((\hat{f})^{-n}(y)\right)$ has a limit point $y_{0}$. Then $\pi\left(y_{0}\right)$ is a limit point of $\left(x^{-n}\right)$, so $\pi\left(y_{0}\right) \in \Omega_{j}$. Since $y_{0} \in$ $\Omega(\hat{f})$, we must have $y_{0} \in \hat{\Omega}_{j}$. Hence $y \in W^{u}\left(\hat{\Omega}_{j}\right)$. By exactly the same argument it follows that $y \in W^{s}\left(\hat{\Omega}_{i}\right)$. Hence $\hat{\Omega}_{i}<\hat{\Omega}_{j}$. Q.E.D.

THEOREM D. Let $f \in \operatorname{End}(M)$ with all singularities of $f$ in the stable manifolds of sinks. Suppose $f$ satisfies Axiom A and $\Omega(f)$ consists of expanding basic sets, together with finitely many sinks. Then $f$ is $\Omega$-stable.

Proof. Let $N$ be a tubular neighborhood of $M$, and let $\hat{f}$ be constructed as in Theorem A. Note that $f$ automatically satisfies the no-cycle property. Hence $\hat{f}$ satisfies Axiom A and the no-cycle property, by Theorem A and Lemma 2.

By the $\Omega$-stability theorem for diffeomorphisms [10] there is a neighborhood $N(\hat{f})$ in $\operatorname{End}(N)$ such that $\hat{g} \in N(\hat{f})$ implies:

(1) $\hat{g}$ is $\Omega$-conjugate to $f$,

(2) $\hat{g}$ satisfies Axiom $A$ and the no-cycle property, and

(3) $\hat{g}$ has expanding and contracting directions corresponding to those of $\hat{f}$.

Pick a neighborhood $N(f)$ of $f$ in $\operatorname{End}(M)$ such that $g \in N(f)$ implies $\hat{g} \in N(\hat{f})$, for some diffeomorphism $\hat{g}$ obtained from $g$ as in Theorem A.

Let $g \in N(f)$. We will show $g$ is $\Omega$-conjugate to $f$. Let $\hat{h}: \Omega(\hat{f}) \rightarrow \Omega(\hat{g})$ be a conjugacy. 
It follows by construction of $\hat{f}$, and our hypothesis that two points in $\Omega(\hat{f})$ are on the same stable manifold if and only if they are on the same fiber. (By definition, two points $x$ and $y$ in $\Omega(\hat{f})$ are on the same stable manifold if and only if $d\left((\hat{f})^{n}(x),(\hat{f})^{n}(y)\right) \rightarrow 0$ as $n \rightarrow \infty$. See [5] or [9] for some discussion of stable manifolds and the generalized stable manifold theorem.)

Since $\hat{h}$ (being a conjugacy) preserves stable manifolds it follows that $z_{1}$ and $z_{2}$ in $\Omega(\hat{f})$ are on the same fiber if and only if $\hat{h}\left(z_{1}\right)$ and $\hat{h}\left(z_{2}\right)$ are on the same fiber. Hence the map $h: \Omega(f) \rightarrow \Omega(g)$ defined by $h(x)=\pi\left(\hat{h}\left(\pi^{-1}(x)\right)\right.$ ) (where we choose any $\pi^{-1}(x)$ in $\left.\Omega(\hat{f})\right)$ is well defined.

First we show $h$ is continuous. Let $\left(x_{n}\right)$ be a sequence of points in $\Omega(f)$ such that $x_{n} \rightarrow x$ as $n \rightarrow \infty$. For each $n>0$, choose $\hat{x}_{n} \in \Omega(\hat{f})$ such that $\pi\left(\hat{x}_{n}\right)=x_{n}$. Then the sequence $\left(\hat{x}_{n}\right)$ must have a limit point $\hat{x} \in \Omega(\hat{f})$ (since $\Omega(\hat{f})$ is closed). Clearly $\pi(\hat{x})=x$. By continuity of $\hat{h}, \hat{h}(\hat{x})$ is a limit point of $\left(\hat{h}\left(\hat{x}_{n}\right)\right)$. Hence $\pi(\hat{h}(\hat{x}))$ is a limit point of $\left(\pi\left(\hat{h}\left(\hat{x}_{n}\right)\right)\right)$. But $h(x)=\pi(\hat{h}(\hat{x}))$ and $h\left(x_{n}\right)=\pi\left(\hat{h}\left(\hat{x}_{n}\right)\right)$, so $h(x)$ is a limit point of the sequence $\left(h\left(x_{n}\right)\right)$. Hence $h$ is continuous.

Since $\hat{h}$ maps points on different fibers to points on different fibers it follows that $h$ is one-to-one. $h$ is also onto since if $y \in \Omega(g)$ and $x=$ $\pi\left((\hat{h})^{-1}\left(\pi^{-1}(y)\right)\right)$ (where we take any choice of $\pi^{-1}(y)$ in $\left.\Omega(\hat{g})\right)$ then $h(x)=y$. Hence $h$ is a homeomorphism.

Finally let $x \in \Omega(f)$. Pick $\hat{x} \in \Omega(\hat{f})$ such that $\pi(\hat{x})=x$. Then $\pi(\hat{f}(\hat{x}))=$ $f(x)$. Hence $h(f(x))=\pi(\hat{h}(\hat{f}(\hat{x})))=\pi(\hat{g}(\hat{h}(\hat{x})))=g(\pi(\hat{h}(\hat{x})))=g(h(x))$. This shows that $h$ is a conjugacy and completes the proof that $f$ is $\Omega$-stable. Q.E.D.

6. An example. We conclude by giving an example of an endomorphism $f$ and the associated diffeomorphism $\hat{f}$. I would like to thank Candy Block for doing the drawing.

The example is pictured in Figure 2. We have drawn the image $f\left(S^{1}\right)$ inside $S^{1}$, but $f$ is a mapping from $S^{1}$ onto itself. Here $e$ is an expanding fixed point of $f, c$ is a contracting fixed point of $f$, and $s_{1}$ and $s_{2}$ are singularities. Also, $f(k)=$ $f(l)=e, f\left(k^{-1}\right)=k$ and $f\left(c^{-1}\right)=c$. Furthermore, the interval $(k, e)$ is in the stable manifold of $c$, where we use the notation $(a, b)$ to denote the arc from $a$ counterclockwise to $b$.

Since $f\left(k^{-1}, l\right)=(k, e)$, the interval $\left(k^{-1}, l\right)$ is in the stable manifold of $c$. Hence both of the singularities of $f$ are in the stable manifold of $c$, so the hypothesis of Theorem A is satisfied.

Since the map $f$ happens to be of degree one, we may let $N=S^{1} \times I$ (as indicated in Figure 2 by the dotted lines) rather than $N=S^{1} \times D^{2}$ (as we would have to do in general). We perturb $f$ to an immersion $f_{1}: S^{1} \rightarrow N$, where $f_{1}$ may be just the map actually pictured in Figure 2 . Then we extend $f_{1}$ to a diffeomor- 
phism $\hat{f}: N \rightarrow N$. We can make $\hat{f}$ fiber preserving except near the singularities (as indicated in Figure 2), where it is impossible to do so.

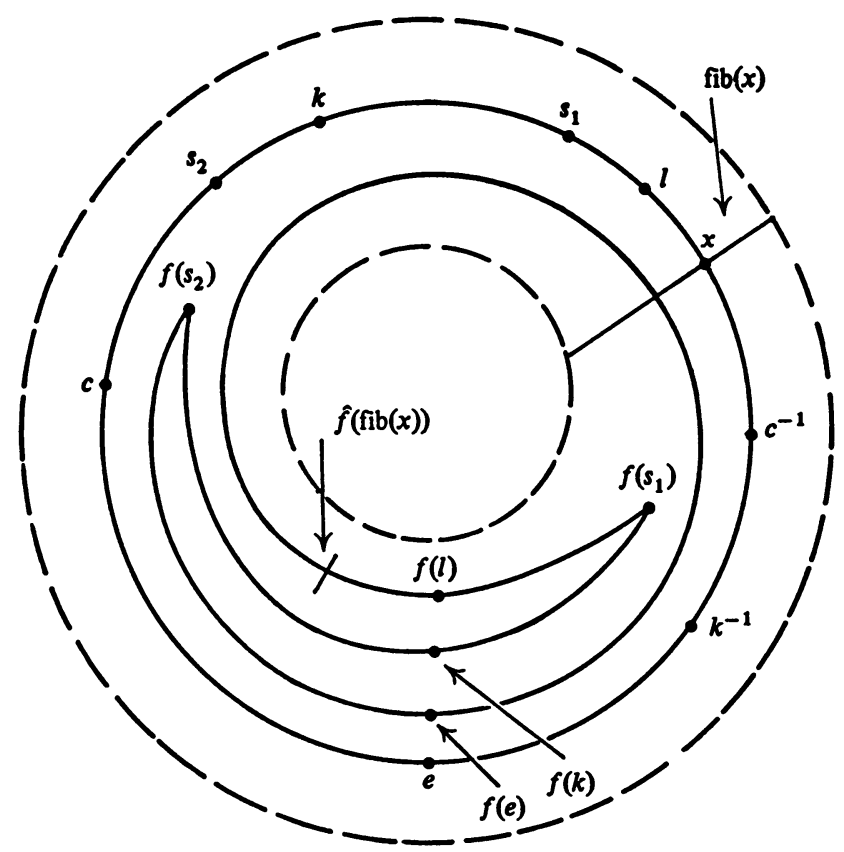

FiguRE 2

Guckenheimer in Proposition 6.1 of [4] says to construct $\hat{f}$ to be fiber preserving on all of $N$, and the remainder of his proof depends on this. This is not possible when folds are present.

\section{REFERENCES}

1. L. Block, Morse-Smale endomorphisms of the circle, Proc. Amer. Math. Soc. 48 (1975), 457-463.

2. L. Block and J. Franke, A classification of the structurally stable contracting endomorphisms of $S^{1}$, Proc. Amer. Math. Soc. 36 (1972), 597-602. MR 46 \#8264. 69-73.

3. - Existence of periodic points for maps of $S^{1}$, Invent. Math. 22 (1973),

4. J. Guckenheimer, Endomorphisms of the Riemann sphere, Proc. Sympos. Pure Math., vol. 14, Amer. Math. Soc., Providence, R.I., 1970, pp. 95-123. MR 43 \#500.

5. M. W. Hirsch and C. C. Pugh, Stable manifolds and hyperbolic sets, Proc. Sympos. Pure Math., vol. 14, Amer. Math. Soc., Providence, R.I., 1970, pp. 133-163. MR 42 \#6872.

6. M. V. Jakobson, On smooth mappings of the circle into itself, Mat. Sb. 85 (127) (1971), 163-188 = Math. USSR Sb. 14 (1971), 161-185. MR 44 \#7587.

7. J. Milnor, Lectures on the h-cobordism theorem, Notes by L. Siebenmann and J. Sondow, Princeton Univ. Press, Princeton, N.J., 1975.

8. M. Shub, Endomorphisms of compact differentiable manifolds, Amer. J. Math. 91 (1969), 175-199. MR 39 \#2169. 
9. S. Smale, Differentiable dynamical systems, Bull. Amer. Math. Soc. 73 (1967), 747-817. MR 37 \#3598; erratum, 39, p. 1593.

10. - The $\Omega$-stability theorem, Proc. Sympos. Pure Math., vol. 14, Amer. Math. Soc., Providence, R.I., 1970, pp. 289-297. MR 42 \#6852.

11. R. F. Williams, One-dimensional non-wandering sets, Topology 6 (1967), 473-487. MR 36 \#897.

DEPARTMENT OF MATHEMATICS, UNIVERSITY OF FLORIDA, GAINESVILLE, FLORIDA 32611 